\title{
Polarizing the Medium: Fermion-Mediated Interactions between Bosons
}

\author{
Dong-Chen Zheng, ${ }^{1,2}$ Lin Wen, ${ }^{3}$ Chun-Rong Ye, ${ }^{1,2}$ and Renyuan Liao ${ }^{1,2, *}$ \\ ${ }^{1}$ Fujian Provincial Key Laboratory for Quantum Manipulation and New Energy Materials, \\ College of Physics and Energy, Fujian Normal University, Fuzhou 350117, China \\ ${ }^{2}$ Fujian Provincial Collaborative Innovation Center for Advanced High-Field \\ Superconducting Materials and Engineering, Fuzhou, 350117, China \\ ${ }^{3}$ College of Physics and Electronic Engineering, Chongqing Normal University, Chongqing 401331, China
}

(Dated: July 10, 2020)

\begin{abstract}
We consider a homogeneous mixture of bosons and polarized fermions. We find that long-range and attractive fermion-mediated interactions between bosons have dramatic effects on the properties of the bosons. We construct the phase diagram spanned by boson-fermion mass ratio and bosonfermion scattering parameter. It consists of stable region of mixing and unstable region toward phase separation. In stable mixing phase, the collective long-wavelength excitations can either be well-behaved with infinite lifetime or be finite in lifetime suffered from the Landau damping. We examine the effects of the induced interaction on the properties of weakly interacting bosons. It turns out that the induced interaction not only enhances the repulsion between the bosons against collapse but also enhances the stability of the superfluid state by suppressing quantum depletion.
\end{abstract}

Ultracold atoms offer fascinating opportunities for investigating quantum many-body problems that relevant to fields as diverse as condensed matter physics, statistical physics, quantum chemistry, and high energy physics [1, 2]. Of particular interest is BoseFermi mixtures [3], which allows one to explore the intriguing physics associated with the interplay between atoms of different quantum statistics. On the experimental side, tremendous progress have been achieved, which include controlling and characterizing the interspecies interactions [4-12], realizing mixture of Bose and Fermi superfluids [13-17], and probing physics of the phase separation state $[18,19]$. On the theoretical side, intense attentions have been paid to study groundstate properties [20-26], nature of excitations [26-30], boson-mediated fermionic superfluidity [31-35], collective dynamics [36, 37], and formation of exotic quantum phases [38-44].

Very recently, adding to the new excitements are the observations of fermion-mediated long-range interactions between bosons [45-47]. The long-range nature of these mediated interactions enriches the toolbox for controlling coherent interactions [48] and opens up the possibility of correlating distant atoms and preparing new quantum phases $[38,49]$. There have been theoretical attempts [50-52] for understanding such fermion-mediated interactions based on the linear response theory. Given current experimental relevance, thorough theoretical understanding and identifying new features arising from fermion-mediated interactions becomes an urgent task.

In this work, we shall carry out a comprehensive study on the fermion-mediated interactions in Bose-Fermi mixtures, with the aim of laying down a solid and tractable framework to treat such problems, fully characterizing the mediated interactions, and elucidating the effects of the induced interactions on the bosons. First, we shall start from the functional representation of the partition function of the system. By tracing out the fermionic degrees of freedom, we obtain an effective action solely in terms of bosonic degrees of freedom, so that we can isolate the mediated effects of the fermions on the bosons. Second, we will examine the induced interaction at static limit in order to obtain an effective interaction potential. Third, we construct a phase diagram by taking account of both phase stability and the Landau damping of Bogoliubov excitations arising from density response from the Fermi gases. Finally, we examine the quantum fluctuations in the presence of the effective potential on the properties of the bosons.

We consider a homogeneous mixture of Bose gases and spin-polarized Fermi gases, described by the following grand canonical Hamiltonian

$$
\begin{aligned}
H & =H_{B}+H_{F}+H_{I}, \\
H_{B} & =\int d \mathbf{r} \phi^{\dagger}(\mathbf{r})\left(-\frac{\hbar^{2} \nabla^{2}}{2 m_{B}}-\mu_{B}\right) \phi(\mathbf{r}), \\
H_{F} & =\int d \mathbf{r} \psi^{\dagger}(\mathbf{r})\left(-\frac{\hbar^{2} \nabla^{2}}{2 m_{F}}-\mu_{F}\right) \psi(\mathbf{r}), \\
H_{I} & =\int d \mathbf{r}\left(g_{I} \psi^{\dagger} \psi \phi^{\dagger} \phi+\frac{g}{2} \phi^{\dagger} \phi^{\dagger} \phi \phi\right) .
\end{aligned}
$$

For bosons, $\phi(\mathbf{r})$ is the field operator, $m_{B}$ is the mass of an atom, and $\mu_{B}$ is the chemical potential. For fermions, $\psi(\mathbf{r})$ is the field operator, $m_{F}$ is the mass of an atom, and $\mu_{F}$ is the chemical potential. In the interaction term $H_{I}$, the coupling $g_{I}=2 \pi \hbar^{2} a_{F B}\left(m_{F}^{-1}+m_{B}^{-1}\right)$ accounts for the interactions between the fermions and the bosons, and $g=4 \pi \hbar^{2} a_{B B} / m_{B}>0$ accounts for the repulsive interactions between bosons, where $a_{F B}$ and $a_{B B}$ are the corresponding s-wave scattering lengths. For convenience, we define the Fermi momentum $k_{F}=\left(6 \pi^{2} n_{F}\right)^{1 / 3}$ with $n_{F}$ being the number density of Fermi gases, the Fermi velocity $v_{F}=\hbar k_{F} / m_{F}$ and the corresponding Fermi energy $E_{F}=\hbar^{2} k_{F}^{2} / 2 m_{F}$. We shall take natural units by setting $\hbar=k_{B}=1$ for sake of simplicity from now on. 
Within the framework of the imaginary-time field integral, the partition function of the system can be cast as $\mathcal{Z}=\int d[\bar{\psi}, \psi] d\left[\phi^{*}, \phi\right] e^{-S}$ with the action given by [53] $S=\int_{0}^{\beta} d \tau\left[H+\int d^{3} \mathbf{r}\left(\bar{\psi} \partial_{\tau} \psi+\phi^{*} \partial_{\tau} \phi\right)\right]$, where $\beta=1 / T$ is the inverse temperature. Carrying out the integration over the fermionic degrees of freedom, we obtain an effective action solely in terms of bosonic degrees of freedom $S_{\text {eff }}=S_{B}-\operatorname{Tr} \ln \mathcal{M}$, where

$$
\begin{aligned}
S_{B} & =\int d \tau d \mathbf{r}\left[\phi^{*}\left(\partial_{\tau}-\frac{\nabla^{2}}{2 m_{B}}-\mu_{B}\right) \phi+\frac{g}{2}\left(\phi^{*} \phi\right)^{2}\right], \\
\mathcal{M} & =\partial_{\tau}-\frac{\nabla^{2}}{2 m_{F}}-\mu_{F}+g_{I} \phi^{*} \phi .
\end{aligned}
$$

Up to this level, the formal manipulation of the partition function is exact. To distill low-energy physics, we shall resort to some sorts of approximations to be elaborated on.

To proceed, we may write $\phi^{*} \phi=\rho_{0}+\sum_{q \neq 0} \rho_{q} e^{i q x}$, and we set $\mathcal{M}=-\mathcal{G}^{-1}+\mathcal{M}_{1}$ where $\mathcal{G}^{-1}=-\partial_{\tau}+$ $\nabla^{2} / 2 m_{F}+\mu_{F}-g_{I} \rho_{0}$ is the inverse fermionic Green's function and $\mathcal{M}_{1}=g_{I} \sum_{q \neq 0} \rho_{q} e^{i q x}$, with $x$ being spacetime coordinate. This allows one to write $\operatorname{Tr} \ln \mathcal{M}=$ $\operatorname{Tr} \ln \left(-\mathcal{G}^{-1}\right)+\operatorname{Tr} \ln \left(1-\mathcal{G M}_{1}\right)$ and to perform series expansions as follows

$$
-\operatorname{Tr} \ln \left(1-\mathcal{G M}_{1}\right)=\sum_{l=1} \frac{1}{l} \operatorname{Tr}\left[\left(\mathcal{G M}_{1}\right)^{l}\right] .
$$

To fully exploit the translational invariance of the system, we shall transform the above to momentum-frequency representation $\left(q \equiv\left(\mathbf{q}, i w_{m}\right)\right)$ resulting in

$$
\begin{aligned}
& \operatorname{Tr}\left(\mathcal{G M}_{1}\right)=\mathcal{M}_{1}(0) \sum_{\left(\mathbf{k}, i w_{n}\right)} \mathcal{G}(k)=0, \\
& \frac{1}{2} \operatorname{Tr}\left[\left(\mathcal{G M}_{1}\right)^{2}\right]=\beta V \frac{g_{I}^{2}}{2} \sum_{q \neq 0} \Pi_{q} \rho_{q} \rho_{-q}, \\
& \Pi_{q}=\frac{1}{\beta V} \sum_{\left(\mathbf{k}, i w_{n}\right)} \mathcal{G}(k) \mathcal{G}(k+q) .
\end{aligned}
$$

Several comments are in order: For the expansion in Eq. (3), the $l=1$ term vanishes, as can be seen from Eq. (4a); The $l=2$ term corresponds to induced twobody interactions between bosons, as can be seen from Eq. (4b) and Eq. (4c), where we have defined the so-called polarization function $\Pi_{q} ; V$ is the volume of the system, $w_{n}=\pi(2 n+1) / \beta$ is fermionic Matsubara frequencies, while $w_{m}=2 \pi n / \beta$ is the bosonic Matsubara frequencies, where $n$ 's are integers; We will neglect $l>=3$ terms, as they represent induced three-body or more than threebody interactions for bosons, which are usually irrelevant for dilute gases. To be concrete, the effective action for the system is approximated as

$$
S_{e f f}=S_{B}-\operatorname{Tr} \ln \left(-\mathcal{G}^{-1}\right)+\beta V \frac{g_{I}^{2}}{2} \sum_{q \neq 0} \Pi_{q} \rho_{q} \rho_{-q} .
$$

We follow the standard Bogoliubov decomposition by splitting the bosonic field $\phi$ into a mean-field part $\phi_{0}$ and a fluctuating part $\varphi: \phi=\phi_{0}+\varphi$. By retaining the fluctuating fields up to the quadratic order, we approximate the effective action as $S_{\text {eff }} \approx S_{0}+S_{g}$, where $S_{0}$ is the mean-field action and $S_{g}$ is the gaussian action with quadratic orders of the fluctuating fields $\varphi_{q}^{*}$ and $\varphi_{q}$. Employing $\Omega=-\ln \mathcal{Z} / \beta V$, we obtain that the grand potential density at the mean-field level $\Omega^{(0)}=S_{0} / \beta V$ becomes

$$
\Omega^{(0)}=\frac{g\left|\phi_{0}\right|^{4}}{2}-\mu_{B}\left|\phi_{0}\right|^{2}-\frac{1}{\beta V} \sum_{\mathbf{k}} \ln \left(1+e^{-\beta \xi_{\mathbf{k}}}\right),
$$

where $\xi_{\mathbf{k}}=\mathbf{k}^{2} / 2 m_{F}-\mu_{F}+g_{I}\left|\phi_{0}\right|^{2}$.

Minimization of $\Omega^{(0)}$ with respect to the condensate order parameter $\phi_{0}^{*}$ leads to the Hugenholz-Pines theorem [54] determining the chemical potential $\mu_{B}=$ $g_{I} n_{F}+g\left|\phi_{0}\right|^{2}$. Without loss of generality, we shall take $\phi_{0}=\sqrt{n_{B}}$, where $n_{B}$ is the condensate density of the Bose gases. The self-consistent condition for the fermion density is determined via $n_{F}=-\partial \Omega^{(0)} / \partial \mu_{F}$, yielding

$$
n_{F}=\frac{1}{V} \sum_{\mathbf{k}} f\left(\xi_{\mathbf{k}}\right),
$$

where $f(x)=1 /[1+\exp (\beta x)]$ is the Fermi-Dirac distribution function. Solving the above equation we obtain the chemical potential for the Fermi gases: $\mu_{F}=E_{F}+g_{I} n_{B}$.

At the mean-field level, the ground-state energy density can be obtained via $E_{G}^{(0)}=\Omega^{(0)}+\mu_{F} n_{F}+\mu_{B} n_{B}$, yielding

$$
E_{G}^{(0)}=\frac{3}{5} n_{F} E_{F}+\frac{g}{2} n_{B}^{2}+g_{I} n_{F} n_{B} .
$$

For the system to be stable, we naturally require that the Hessian matrix $\partial^{2} E_{G}^{(0)} / \partial n_{i} \partial n_{j}(i, j=F, B)$ constructed for the ground-state energy $E_{G}\left(n_{F}, n_{B}\right)$ to be positively definite, which leads to an upper bound for the fermion density

$$
n_{F}^{1 / 3}<\frac{g}{3 m_{F} g_{I}^{2}}\left(6 \pi^{2}\right)^{2 / 3},
$$

which gives the condition for mechanical stability of the system [31].

As seen from the effective action in Eq. (5), the Hamiltonian describing induced two-body interactions between bosons through coupling with fermions is given by

$$
H_{i n d}=\frac{g_{I}^{2}}{2} \sum_{\mathbf{q} \neq 0} \sum_{\mathbf{k}, \mathbf{p}} \Pi_{\mathbf{q}} \phi_{\mathbf{k}+\mathbf{q}}^{\dagger} \phi_{\mathbf{p}-\mathbf{q}}^{\dagger} \phi_{\mathbf{p}} \phi_{\mathbf{k}} .
$$

Here, $\Pi_{\mathbf{q}} \equiv \Pi_{(\mathbf{q}, 0)}$ is the polarization function evaluated at the static limit at zero temperature, which reads

$$
\Pi_{\mathbf{q}}=-\frac{d\left(E_{F}\right)}{4}\left[1+\frac{k_{F}^{2}-q^{2} / 4}{k_{F} q} \ln \left|\frac{q+2 k_{F}}{q-2 k_{F}}\right|\right] .
$$



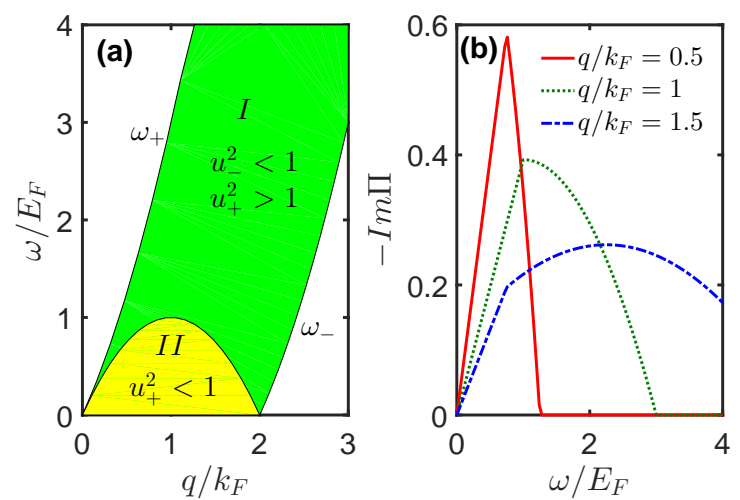

FIG. 1. (color online) (a) The shade region is the range where the imaginary part of the polarization function differs from zero, and it is referred as particle-hole continuum, since it is the region of single-particle excitations, whereby a particle below the Fermi surface is excited to above the Fermi surface. Outside this region, it is not possible to conserve energy and wave vector in a single-particle excitation process. (b) The imaginary part of the polarization function $\operatorname{Im} \Pi(\mathbf{q}, \omega)$ [in units of $d\left(E_{F}\right)$ ] as a function of frequencies $\omega$ at given different typical momentum amplitude $q$.

where $d\left(E_{F}\right)=m_{F} k_{F} / \pi^{2}$ is the density of states at the Fermi energy.

This corresponds to an induced pairwise interaction potential between two Bose atoms with relative coordinate $\mathbf{r}$, given by

$$
\begin{aligned}
V_{\text {ind }}(\mathbf{r}) & =-\frac{d\left(E_{F}\right) g_{I}^{2}}{4} V_{R K K Y}(r), \\
V_{R K K Y}(r) & =\frac{\sin \left(2 k_{F} r\right)-2 k_{F} r \cos \left(2 k_{F} r\right)}{2 \pi k_{F} r^{4}} .
\end{aligned}
$$

The induced attractive long-range interaction is of the RKKY type [55] in real space, where it decays at $1 / r^{3}$ and shows the Friedel oscillations at a wave vector $2 k_{F}$.

The gaussian action for the bosonic fluctuating fields can be written as $S_{g}=\frac{1}{2} \sum_{q} \Phi_{q}^{\dagger} \mathcal{G}_{B}^{-1}(q) \Phi_{q}$ with $\Phi_{q}=$ $\left(\varphi_{q}, \varphi_{-q}^{*}\right)^{T}$ and the matrix $\mathcal{G}_{B}^{-1}(q)$ given by

$$
\mathcal{G}_{B}^{-1}(q)=\left(\begin{array}{cc}
-i w_{m}+\epsilon_{\mathbf{q}}+A_{q} & A_{q} \\
A_{q} & i w_{m}+\epsilon_{\mathbf{q}}+A_{q}
\end{array}\right),
$$

where $\epsilon_{\mathbf{q}}=\mathbf{q}^{2} / 2 m_{B}$ and $A_{q}=\left(g+g_{I}^{2} \Pi_{q}\right) n_{B}$. The quasiparticle spectrum $\omega(\mathbf{q})$ and the damping rate $\gamma(\mathbf{q})$ can be obtained by seeking solutions of the secular equation $\operatorname{det}_{B}^{-1}(\mathbf{q}, \omega-i \gamma)=0$ with substitution of $\Pi_{q} \mid i w_{m} \rightarrow$ $\omega+i 0^{\dagger}$. The real part of polarization function $\Pi_{q}$ determines the shift of the spectrum while the imaginary part gives rise to the damping of the excitations.

By analytic continuation to real frequency $(i \omega \rightarrow \omega+$ $i 0^{\dagger}$ ), one obtains the real part and the imaginary part of the polarization function, so-called the Lindhard function $[56]$

$$
\begin{aligned}
& \operatorname{Re} \Pi(\mathbf{q}, \omega)=-\frac{d\left(E_{F}\right)}{4}\left[1-\frac{1-u_{-}^{2}}{2 q / k_{F}} \ln \left|\frac{1+u_{-}}{1-u_{-}}\right|+\frac{1-u_{+}^{2}}{2 q / k_{F}} \ln \left|\frac{1+u_{+}}{1-u_{+}}\right|\right], \\
& \operatorname{Im} \Pi(\mathbf{q}, \omega)=-d\left(E_{F}\right) \frac{\pi k_{F}}{8 q}\left[\left(1-u_{-}^{2}\right) \Theta\left(1-u_{-}^{2}\right)-\left(1-u_{+}^{2}\right) \Theta\left(1-u_{+}^{2}\right)\right],
\end{aligned}
$$

where $u_{ \pm}=\omega / q v_{F} \pm q / 2 k_{F}$. Physically, it describes the response of the Fermi gases under external density perturbations exerted by the Bose gases. The imaginary part of the polarization function provides essential information for the damping of excitations, as it can be related to the dynamical structure factor through the fluctuationdissipation theorem [57]. From Eq. (14b), we find that it has contributions from two situations enforced by Diracdelta function: one is for $u_{-}^{2}<1$, and the other is from $u_{+}^{2}<1$. We show the region in momentum-frequency space where the imaginary part of the polarization function $\operatorname{Im} \Pi(\mathbf{q}, \omega)$ differs from zero in Fig. 1(a). In the plot, region I is defined as $u_{-}^{2}<1$ and $u_{+}^{2}>1$, while region II satisfies $u_{+}^{2}<1$. The upper (lower) bound is given by $\omega_{ \pm} / E_{F}=\left(q / k_{F}\right)^{2} \pm 2 q / k_{F}$. In panel (b), we plot $I m \Pi$ as a function of frequencies $\omega$ for three typical momenta amplitude $q / k_{F}=0.5,1.0,1.5$. The curve will be linear when the corresponding $(\mathbf{q}, \omega)$ lying in region
II, where both terms in Eq. (14b) contribute. Otherwise, the curve will be parabolic when the corresponding $(\mathbf{q}, \omega)$ lying in region I, where only the first term in Eq. (14b) contributes.

With the information of the polarization function at hand, it is straightforward to obtain the quasiparticle spectrum $\omega(\mathbf{q})$ and the damping rate $\gamma(\mathbf{q})$, shown in Fig. 2. From Eq. (14b), one obtains that the region for damping to occur is given by the inequality constraint $\left(q / k_{F}\right)^{2}-2 q / k_{F}<\omega / E_{F}<\left(q / k_{F}\right)^{2}+2 q / k_{F}$, as shown in the shade region on panel (a) in Fig. 2. At small momenta, the spectrum is phonon-like with the sound velocity given by $c=\sqrt{\left(g+g_{I}^{2} \Pi_{0}\right) n_{B} / m_{B}}$. The positivity of the sound velocity yields the stability constraint $\left(k_{F} a_{F B}\right)^{2}<2 \pi k_{F} a_{B B} m_{F} m_{B} /\left(m_{F}+m_{B}\right)^{2}$, coincident to the mechanical stability provided in Eq. (9). The mass of bosons $m_{B}$ has dramatic effects on the spectrum: at low momenta, the slope is inversely proportional to $\sqrt{m_{B}}$; 

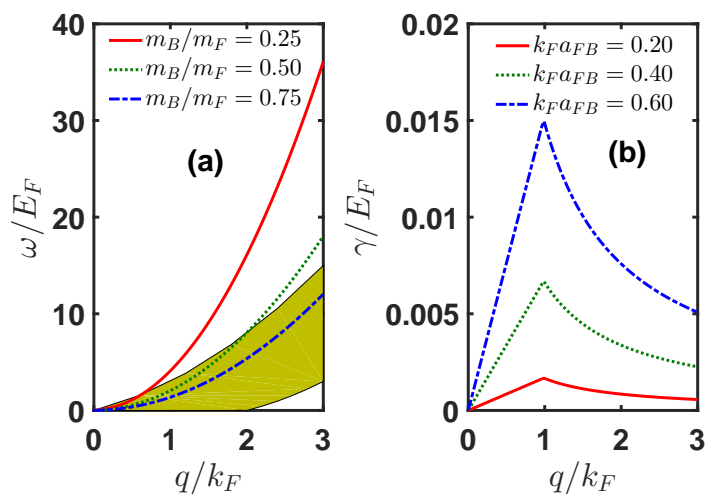

FIG. 2. (color online) Properties of the Bogoliugov quasiparticles: (a) the excitation energy $\omega / E_{F}$ where $k_{F} a_{F B}=0.3$ and (b) the Landau damping rate $\gamma / E_{F}$ where $m_{B} / m_{F}=1$. The shade region is referred as particle-hole continuum. The quasi-particle spectrum laying outside of the shadow region is well-defined, being immune from the Landau damping. We set $k_{F} a_{B B}=0.3$ and $n_{B} / n_{F}=0.2$.

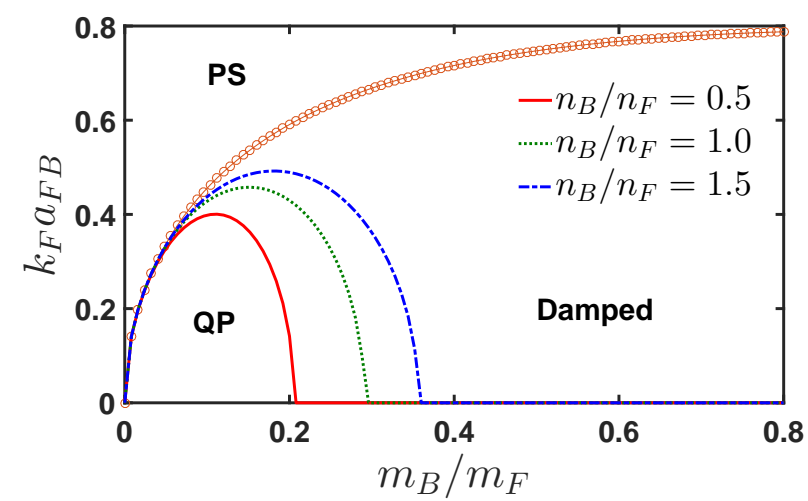

FIG. 3. (color online) Phase diagram spanned by mass ratio $m_{B} / m_{F}$ and interspecies coupling strength $k_{F} a_{F B}$. PS stands for phase separation and QP stands for quasi-particle with infinite lifetime. Here we set $k_{F} a_{B B}=0.4$, which sets bosons in a weakly-interacting regime.

while at high momenta, it gives the mass for free particle. For sufficiently small $m_{B} / m_{F}$, the excitations can achieve infinite lifetime. The damping of the excitations show sharp peak at wave-vector $q=k_{F}$ for all three typical boson-fermion scattering parameter $k_{F} a_{F B}$.

We are now in a position to construct a phase diagram for the system. The stability constraint marks the transition line between stable mixing phase and phase separation (PS) into fermions and bosons [20, 21, 23], shown in Fig. 3, which stays intact for different number density ratio. In the stable region, we can further classify it into quasiparticle excitations with infinite lifetime and with finite lifetime due to the Landau damping, by which a particle absorbs an excitation of momentum $\hbar \mathbf{q}$ and energy $\hbar \omega$ to allow it to move from beneath to
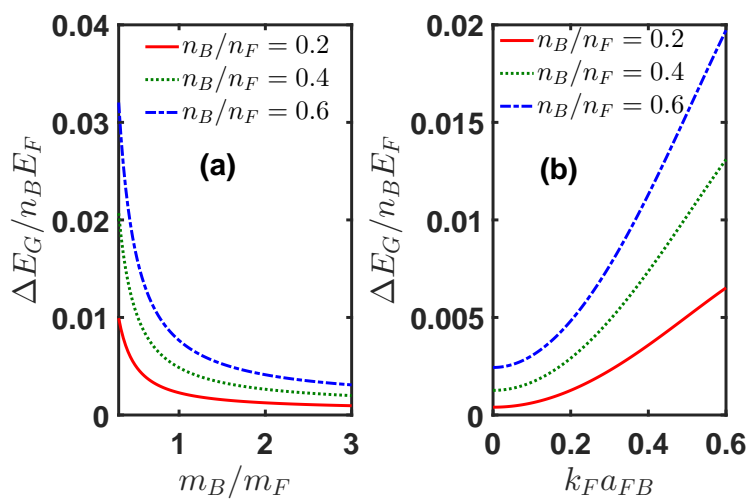

FIG. 4. (color online) Correction to the ground-state energy per density $\Delta E_{G} / n_{B} E_{F}$ (a) as a function of mass ratio $m_{B} / m_{F}$ where $k_{F} a_{F B}=0.3$ and (b) as a function of bosonfermion scattering length $k_{F} a_{F B}$ where $m_{B} / m_{F}=1$. We set $k_{F} a_{B B}=0.3$.

above the Fermi surface, creating a particle-hole pair. To search for well-defined, long-lived excitations, we consider the region where $\operatorname{Im} \Pi(\mathbf{q}, \omega)=0$. This occurs when $\omega / q v_{F}>1+q / 2 k_{F}$ [see Eq. (14b)]. At long-wavelength, this becomes $c / E_{F}>2 / k_{F}$, yielding

$$
\left(k_{F} a_{F B}\right)^{2}<\frac{2 \pi m_{F} m_{B}}{\left(m_{F}+m_{B}\right)^{2}}\left(k_{F} a_{B B}-\frac{3 \pi}{2} \frac{n_{F}}{n_{B}} \frac{m_{B}^{2}}{m_{F}^{2}}\right) .
$$

The phase diagram constructed is shown in Fig. 3. The region of the quasiparticle excitations with infinite lifetime (QP) gets expanded by tuning up the number density ration $n_{B} / n_{F}$. It should be pointed out that we focus on the Landau damping of the collective long-wavelength excitation, where Beliaev damping is strongly suppressed at low momenta [58].

To examine the effects of the effective potential upon the Bose gases, we shall evaluate the ground-state energy correction arising from quantum fluctuations. The fluctuation correction to the thermodynamic potential is given by $\Omega_{f}=\frac{\beta}{2} \operatorname{Tr} \ln \mathcal{G}_{B}^{-1}-\sum_{\mathbf{q}}\left(\epsilon_{\mathbf{q}}+A_{\mathbf{q}}\right)$. At zero temperature, the corresponding ground-state energy correction, becomes renormalized as

$$
\Delta E_{G}=\frac{1}{2} \sum_{\mathbf{q}}\left(\omega_{\mathbf{q}}-\epsilon_{\mathbf{q}}-A_{\mathbf{q}}+\frac{g^{2} n_{B}^{2}}{2 \epsilon_{\mathbf{q}}}\right),
$$

where the Bogoliugov spectrum is given by $\omega_{\mathbf{q}}=$ $\sqrt{\epsilon_{\mathbf{q}}\left(\epsilon_{\mathbf{q}}+A_{\mathbf{q}}\right)}$, and $A_{\mathbf{q}}=\left(g+g_{I}^{2} \Pi_{\mathbf{q}}\right) n_{B}$.

The behavior of fluctuation correction to the groundstate energy $\Delta E_{G}$ is shown in Fig. 4 . In panel (a), we find that as the mass ratio $m_{B} / m_{F}$ increases the energy correction decreases, which is reasonable since the kinetic energy is inversely proportional to the mass of the bosons. It is interesting to notice that increasing the density ratio $n_{B} / n_{F}$ actually contributes to the enhancement of the energy correction. Shown in panel 

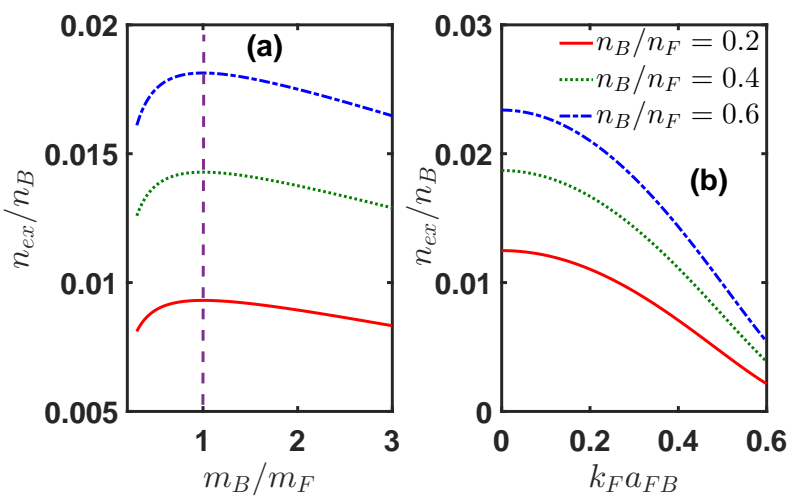

FIG. 5. (color online) Quantum depletion of the condensates $n_{e x} / n_{B}$ (a) as a function of mass ratio $m_{B} / m_{F}$ where $k_{F} a_{F B}=0.3$ and (b) as a function of boson-fermion scattering parameter $k_{F} a_{F B}$ where $m_{B} / m_{F}=1$. The vertical dash line intercepts with maxima of the curves at $m_{B}=m_{F}$. We set $k_{F} a_{B B}=0.3$.

(b), the energy correction increases monotonically with boson-fermion coupling strength $k_{F} a_{F B}$. For vanishing boson-fermion interaction, one can verify that the energy correction recovers the Lee-Huang-Yang correction [59] to the spinless weakling interacting bosons $\Delta E_{G} /\left(g n_{B}^{2}\right)=$ $64 /(15 \sqrt{\pi}) \sqrt{n_{B} a_{B B}^{3}}$. It is remarkable that the correction of energy increases steadily with increasing $k_{F} a_{F B}$. This raises the possibility of realizing quantum droplets states with enhanced quantum repulsion again collapse.

We turn to the depletion of the condensates due to quantum fluctuations, which provides key information about the robustness of the superfluid state. The number of excited particles is evaluated as

$$
n_{e x}=\sum_{q} \mathcal{G}_{B 11}\left(\mathbf{q}, i w_{n}\right)=\sum_{\mathbf{q}} \frac{\epsilon_{\mathbf{q}}+A_{\mathbf{q}}-E_{\mathbf{q}}}{2 E_{\mathbf{q}}} .
$$

The variation of quantum depletion with respect to tuning parameters $m_{B} / m_{F}$ and $k_{F} a_{F B}$ are shown in Fig. 5 for fixed density ratios. Aesthetically appealingly, $n_{e x} / n_{B}$ develops a maximum at equal mass $m_{B}=$ $m_{F}$. Remarkably, increasing the boson-fermion interaction suppresses the quantum depletion, due to attractive nature of induced interaction between bosons. At zero boson-fermion coupling, it recovers the known result [60] for spinless weakly interacting Bosons $n_{e x}=$ $\left(g n_{B}\right)^{3 / 2} /\left(3 \pi^{2}\right)$.

In summary, we find that the induced interaction mediated by fermions between bosons are long-range attractive interactions, tunable with fermion density as well as boson-fermion scattering length. We map out the phase boundary separating stable region of mixing phases and unstable region toward phase separation. We show that the stable region can be further classified by damping of the excitations. The predicted damping rate can be probed experimentally via two-phonon Bragg spec- troscopy [61]. Extension of current work to trapped cases $[3,62,63]$ will facilitate the experimental verification of our predictions. Finally, we analyze the effects of the induced interactions on the ground-state energy correction and quantum depletion of the system. It suggests that by coupling to Fermi gases, weakling interacting bosons may form quantum droplet states $[42,43]$ with enhanced stability. We expect our study contribute to a better understanding of emergent phenomena associated with fermion-mediated interactions.

\section{ACKNOWLEDGMENTS}

R.L. acknowledges funding from the NSFC under Grant No.11674058 and NCET-13-0734. L.W. was supported by NSFC under Grant No. 11875010, and by the Natural Science Foundation of Chongqing under Grant No. cstc2019jcyj-msxmX0217.

* ryliao@fjnu.edu.cn

[1] I. M. Georgescu, S. Ashhab, and F. Nori, Quantum simulation, Rev. Mod. Phys. 86, 153 (2014).

[2] C. Gross and I. Bloch, Quantum simulation with ultracold atoms in optical lattcices, Science 357, 995 (2017).

[3] R. Onofrio, Cooling and thermometry of atomic fermi gases, Physics-Uspekhi 59, 1129 (2016).

[4] G. Modugno, G. Roati, F. Biboli, F. Ferlaino, R. J. Brecha, and M. Inguscio, Collapse of a degenerate fermi gas, Science 297, 2240 (2001).

[5] G. Ferrari, M. Inguscio, W. Jastrzebski, G. Modugno, G. Roati, and A. Simoni, Collisional properties of ultracold k-rb mixtures, Phys. Rev. Lett. 89, 053202 (2002).

[6] G. Roati, F. Riboli, G. Modugno, and M. Inguscio, Fermi-bose quantum degenerate k-rb mixture with attractive interaction, Phys. Rev. Lett. 89, 150403 (2002).

[7] C. A. Stan, M. W. Zwierlein, C. H. Schunck, S. M. F. Raupach, and W. Ketterle, Observation of feshbach resonances between two different atomic species, Phys. Rev. Lett. 93, 143001 (2004).

[8] S. Inouye, J. Goldwin, M. L. Olsen, C. Ticknor, J. L. Bohn, and D. S. Jin, Observation of heteronuclear feshbace resoances in a mixture of bosons and fermions, Phys. Rev. Lett. 93, 183201 (2004).

[9] C. Ospelkaus, S. Ospelkaus, K. Sengstock, and K. Bongs, Interaction-driven dynamics of k-rb fermion-boson gas mixtures in the large-particle-number limit, Phys. Rev. Lett. 96, 020401 (2006).

[10] S. Ospelkaus, C. Ospelkaus, L. Humbert, K. Sengstock, and K. Bongs, Tuning of heteronuclear interations in a degenerate fermi-bose mixture, Phys. Rev. Lett. 97, 120403 (2006).

[11] B. J. DeSalvo, K. Patel, J. Johansen, and C. Chin, Observation of a degenerate fermi gas trapped by a boseeinstein condensate, Phys. Rev. Lett. 119, 233401 (2017).

[12] Y.-X. Ye, L.-Y. Xie, Z. Guo, X.-B. Ma, G.-R. Wang, L. You, and M. K. Tey, Double degenerate bose-fermi mixture of strontium and lithium, arxiv:2006.15654. 
[13] I. Ferrier-Barbut, M. Delehaye, S. Laurent, A. T. Grier, M. Pierce, B. S. Rem, F. Chevy, and C. Salomon, A mixture of bose and fermi superfluids, Science 345, 1035 (2014).

[14] M. Delehaye, S. Laurent, I. Ferrier-Barbut, S. Jin, F. Chevy, and C. Salomon, Critical velocity and dissipation of an ultracold bose-fermi counterflow, Phys. Rev. Lett. 115, 265303 (2015).

[15] X.-C. Yao, H.-Z. Chen, Y.-P. Wu, X.-P. Liu, X.-Q. Wang, X. Jiang, Y. Deng, Y.-A. Chen, and J.-W. Pan, Observation of coupled vortex lattices in a mass-imbalance bose and fermi superfluid mixture, Phys. Rev. Lett. 117, 145301 (2016).

[16] R. Roy, A. Green, R. Bowler, and S. Gupta, Two-element mixture of bose and fermi superfluids, Phys. Rev. Lett. 118, 055301 (2017).

[17] Y.-P. Wu, X.-C. Yao, X.-P. Liu, X.-Q. Wang, Y.-X. Wang, H.-Z. Chen, Y. Deng, Y.-A. Chen, and J.-W. Pan, Coupled dipole oscillations of a mass-imbalanced bosefermi superfluid mixture, Phys. Rev. B 97, 020506(R) (2018).

[18] R. S. Lous, I. Fritsche, M. Jag, F. Lehmann, E. Kirilov, B. Huang, and R. Grimm, Probing the interface of a phase-separated state in a repulsive bose-fermi mixture, Phys. Rev. Lett. 120, 243403 (2018).

[19] B. Huang, I. Fritsche, R. S. Lous, C. Baroni, J. T. M. Walraven, E. Kirilov, and R. Grimm, Breathing mode of a bose-einstein condensate repulsively interacting with a fermionic reservoir, Phys. Rev. A 99, 041602(R) (2019).

[20] K. Mølmer, Bose condensates and fermi gases at zero temperature, Phys. Rev. Lett. 80, 1804 (1998).

[21] L. Viverit, C. J. Pethick, and H. Smith, Zero-temperature phase diagram of binary boson-fermion mixtures, Phys. Rev. A 61, 053605 (2000).

[22] L. Viverit and S. Giorgini, Ground-state properties of a dilute bose-fermi mixture, Phys. Rev. A 66, 063604 (2002).

[23] R. Roth, Struture and stability of trapped atomic bosonfermion mixture, Phys. Rev. A 66, 013614 (2002).

[24] F. M. Marchetti, C. J. M. Mathy, D. A. Huse, and M. M. Parish, phase separation and collapse in bose-fermi mixtures with a feshbach resonance, Phys. Rev. B 78, 134517 (2008).

[25] D. Ludwig, S. Floerchinger, S. Moroz, and C. Wetterich, Quantum phase transition in bose-fermi mixtures, Phys. Rev. A 84, 033629 (2011).

[26] R. Zhang, W. Zhang, H. Zhai, and P. Zhang, Calibration of interaction energy between bose and fermi superfluids, Phys. Rev. A 90, 063614 (2014).

[27] H. Pu, W. Zhang, M. Wilkens, and P. Meystre, Phonon spectrum and dynamical stability of a dilute quantum degenerate bose-fermi mixture, Phys. Rev. Lett. 88, 070408 (2002).

[28] X.-J. Liu and H. Hu, Finite-temperature excitations of a trapped bose-fermi mixture, Phys. Rev. A 68, 033613 (2003).

[29] D. H. Santamore, S. Gaudio, and E. Timmermans, Zero sound in a mixture of a single-component fermion gas and a bose-einstein condensate, Phys. Rev. Lett. 93, 250402 (2004).

[30] J. H. Pixley, X. Li, and S. DasSarma, Damping of longwavelength collective modes in spinor bose-fermi mixtures, Phys. Rev. Lett. 114, 225303 (2015).
[31] H. Heiselberg, C. J. Pethick, H. Smith, and L. Viverit, Influence of induced interactions on the superfluid transition in dilute fermi gases, Phys. Rev. Lett. 85, 2418 (2000).

[32] M. J. Bijlsma, B. A. Heringa, and H. T. C. Stoof, Phonon exchange in dilute fermi-bose mixtures: tailoring the fermi-fermi interaction, Phys. Rev. A 61, 053601 (2000).

[33] K. Suzuki, T. Miyakawa, and T. Suzuki, p-wave superfluid and phase separation in atomic bose-fermi mixtures, Phys. Rev. A 77, 043629 (2008).

[34] Z. Wu and G. M. Bruun, Topological superfluid in a bosefermi mixture with a high critical temperature, Phys. Rev. Lett. 117, 245302 (2016).

[35] J. J. Kinnunen, Z. Wu, and G. M. Bruun, Induced pwave pairing in bose-fermi mixtures, Phys. Rev. Lett. 121, 253402 (2018).

[36] F.Kh.Abdullaev, M. Ogren, and M. P. Sorensen, Collective dynamics of fermi-bose mixtures with an oscillating scattering length, Phys. Rev. A 99, 033614 (2019).

[37] B. Huang, Bose-einstein condensate immersed in a fermi sea: Theory of static and dynamic bevavior across phase separation, Phys. Rev. A 101, 063618 (2020).

[38] H. P. Buchler and G. Blatter, Supersolid versus phase separation in atomic bose-fermi mixtures, Phys. Rev. Lett. 91, 130404 (2003).

[39] S.-K. Tung, K. Jimenez-Garcia, J. Johansen, C. V. Parker, and C. Chin, Geometric scaling of efimov states in a li-cs mixture, Phys. Rev. Lett. 113, 240402 (2014).

[40] Z.-F. Xu, X. Li, P. Zoller, and W. V. Liu, Spontaneous quantum hall effect in an atomic spinor bose-fermi mixture, Phys. Rev. Lett. 114, 125303 (2015).

[41] M. Tylutki, A. Recati, F. Dalfovo, and S. Stringari, Darkbright solitons in a superfluid bose-fermi mixture, New J. Phys. 18, 053014 (2016).

[42] X. Cui, Spin-orbit-coupling-induced quantum droplet in ultracold bose-fermi mixtures, Phys. Rev. A 98, 023630 (2018).

[43] D. Rakshit, T. Karpiuk, M. Brewczyk, M. Lewenstein, and M. Gajda, Self-bound bose-fermi liquids in lower dimensions, New J. Phys. 21, 073027 (2019).

[44] C. Zhu, L. Chen, H. Hu, X. J. Liu, and H. Pu, Spin-exchange-induced exotic superfluids in a bose-fermi spinor mixture, Phys. Rev. A 100, 031602(R) (2019).

[45] B. J. DeSalvo, K. Patel, G. Cai, and C. Chin, Observation of fermion-mediated interactions between bosonic atoms, Nature 568, 61 (2019).

[46] G. M. Bruun, New interactions seen in an ultracold gas, Nature 568, 37 (2019).

[47] H. Edri, B. Raz, N. Matzliah, N. Davidson, and R. Ozeri, Observation of spin-spin fermion-mediated interactions between ultracold atoms, Phys. Rev. Lett. 124, 163401 (2020).

[48] C. Chin, R. Grimm, P. Julienne, and E. Tiesinga, Feshbach resonances in ultracold gases, Rev. Mod. Phys. 82, 1225 (2010).

[49] S. Sachdev, Quantum magnetism and criticality, Nat. Phys. 4, 173 (2008).

[50] D. H. Santamore and E. Timmermans, Fermion-mediated interations in a dilute bose-einstein condenstate, Phys. Rev. A 78, 013619 (2008).

[51] S. De and S. I. B., Fermion-mediated long-range interactions between bosons stored in an optical lattice, Appl. Phys. B 114, 527 (2014). 
[52] J. J. Kinnunen and G. M. Bruun, Induced interactions in a superfluid bose-fermi mixture, Phys. Rev. A 91, 041605(R) (2015).

[53] A. Altland and B. Simons, Condensed Matter Field Theory, 2nd ed. (CUP, Cambridge, UK, 2010).

[54] N. Hugenholz and D. Pines, Ground-state energy and excitation spectrum of a system of interacting bosons, Phys. Rev. 116, 489 (1959).

[55] M. A. Ruderman and C. Kittel, Indirect exchange coupling of nuclear magnetic moments by conduction electrons, Phys. Rev. 96, 99 (1954).

[56] J. Lindhard, On the properties of a gas charged particles, K. Dan. Vidensk. Selsk. Mat. Fys Medd. 28, 8 (1954).

[57] P. Coleman, Introduction to Many-Body Physics (CUP, Cambridge, UK, 2015).

[58] W. V. Liu, Theoretical study of the damping of collective excitations in a bose-einstein condensate, Phys. Rev.
Lett. 79, 4056 (1997).

[59] T. D. Lee, K. Huang, and C. N. Yang, Eigenvalues and eigenfunctions of a bose system of hard spheres and its low-temperature properties, Phys. Rev. 106, 1135 (1957).

[60] N. Nagaosa, Quantum Field Theory in Condensed Matter Physics, 1st ed. (Spinger-Verlag Berlin, Heidelberg, Germany, 1999).

[61] R. Ozeri, N. Katz, J. Steinhauer, and N. Davidson, Colloquium: Bulk bogoliubov excitations in a bose-einstein condensate, Rev. Mod. Phys. 77, 187 (2005).

[62] R. Onofrio and C. Presilla, Reaching fermi degeneracy in two-species optical dipole traps, Phys. Rev. Lett. 89, 100401 (2002).

[63] R.L. acknowledges private communications with Roberto Onofrio regarding trapped cases. 